\title{
Effets in vitro des dermaseptines sur les caractéristiques du sperme
}

\author{
Mounir AJINA ${ }^{1}$, Amira ZAIRI ${ }^{2}$, Soumaya MOUGOU ${ }^{1}$, Samira IBALAA ${ }^{1}$, Radhouène NAIEFER ${ }^{1}$ \\ Ghaya MERDASSI ${ }^{1}$, Mariem MEHDI ${ }^{1}$, Khaled EL HENI², Ali SAAD ${ }^{1}$
}

1 Service de Cytogénétique et de Biologie de la Reproduction, Hôpital Farhat Hached, 2 Laboratoire de Biochimie, Faculté de Médecine Ibn El Jazzar, Sousse, Tunisie

\section{RESUME}

Au cours de ce travail et dans le cadre stratégique du développement des molécules multiactives et plurifonctionnelles, nous nous sommes proposés de tester pour la première fois les dermaseptines (DS), peptides de vertébrés biologiquement actifs, en tant que spermicide.

Nous avons étudié in vitro l'activité de ces nouvelles structures peptidiques, sur la mobilité et la viabilité des spermatozoïdes humains et avons pu montrer que ces DS :

- sont actives à des doses micromolaires contre les spermatozoïdes humains,

- possèdent un spectre d'activité spermicide comparable sinon meilleur que celui du Nonoxynol-9, spermicide classique utilisé actuellement et présentant plusieurs types de contraintes.

Les DS :

- ont un effet dose dépendant,

- ont un mécanisme d'action différent de celui du nonoxynol-9,

- entraînent l'inhibition de la mobilité des spermatozoïdes humains après un temps d'incubation relativement court, suggérant ainsi un mode d'interaction direct du peptide avec la membrane du spermatozoïde,

- ont une parfaite action synergique avec d'autres agents chélateurs tel que I'EDTA qui amplifie considérablement leur activité spermicide et pourraient ainsi pallier au problème de toxicité, d'autant plus que la DS4 présente une relative activité hémolytique.

Ces résultats devraient être exploités d'une manière approfondie, afin de concevoir de nouvelles séquences analogues, dénuées de cytotoxicité et ayant une bioactivité élevée ; ceci devrait ouvrir la voie à la conception d'une nouvelle génération de molécules à spectre d'action large et varié, utilisable comme nouveau spermicide à action plurifonctionnelle.

Mots clés : dermaseptine, spermicide, spermatozoïde

\section{INTRODUCTION}

Les contraceptifs vaginaux chimiques représentent un moyen de contraception efficace lorsqu'ils sont bien utilisés. Ils sont généralement bien tolérés et anodins. Ces contraceptifs méritent de figurer à côté des principales méthodes anticonceptionnelles. L'utilisation contraceptive de produits spermicides est une notion connue depuis des siècles. Les éponges syriennes avaient une réputation qui n'a pas été oubliée. Au siècle dernier, les mignonnettes imbibées de vinaigre avaient une large diffusion.

Vers 1930, de très nombreux spermicides furent proposés dans les pays anglo-saxons, utilisant généralement l'acide borique, l'acide lactique ; leur efficacité était relative et avait nuit à leur réputation. Ces produits sont connus de longue date, mais leur utilisation en tant que spermicide est relativement récente.

Les spermicides sont des produits formés à partir d'une ou plusieurs substances actives. Ils se présentent sous forme de crème, mousse, gel, ovules secs ou moussants, ou tampons. Les substances actives sont des agents bactéricides ou fortement acides, mais surtout des surfactifs.

Ces substances actives sont nombreuses, les plus communes sont : le chlorure de benzalkonium, le Nonoxynol9, l'octoxynol, le menfégal, la cellulose acétate phtalate (CAP), le sodium dodécyl sulfate (SDS) et le PRO-2000.

Les spermicides agissent selon plusieurs mécanismes : coagulation du sperme, immobilisation des spermatozoïdes, altération des spermatozoïdes soit par leur destruction, soit par la perte de leur pouvoir fécondant, altération de la glaire qui devient impénétrable par les spermatozoïdes.

\section{Correspondance :}

Pr Ali SAAD - Laboratoire de Cytogénétique et de Biologie de la Reproduction, CHU Farhat HACHED, 4000, Sousse,

Tunisie - Tel-Fax + 21673219488 -

Email ali.saad@rns.tn 
Les surfactifs agissent essentiellement sur la membrane cellulaire des spermatozoïdes, en abaissant la tension superficielle et provocant un déséquilibre osmotique qui entraîne leur lyse [4].

L'effet contraceptif est renforcé par une action sur la glaire cervicale qui se transforme en un magma gélifié, constituant une véritable barrière dans laquelle s'engluent les spermatozoïdes [17].

Il existe une toute nouvelle famille de peptides antimicrobiens de vertébrés, isolée chez les amphibiens : ce sont les dermaseptines (DS). La famille des DS, baptisées ainsi à cause de leur origine dermique et de leur activité antiseptique [12], regroupe sept peptides de 28 à 34 acides aminés qui possèdent des propriétés biologiques remarquables : cinq dermaseptines $S$ et deux dermaseptines $B$.

Les dermaseptines $S$, au nombre de cinq (DS1, 2, 3, 4, 5), sont une famille de peptides isolés à partir d'extrait de peau de Phyllomedusa sauvagei, une grenouille arboricole sud américaine (Guyane) $[3,11]$ Les dermaseptines B (B1 et B2) sont deux autres peptides isolées à partir de Phyllomedusa bicolor [11].

L'analyse des propriétés pharmacologiques des DS montre que celles-ci sont douées d'une activité antimicrobienne contre les bactéries, les levures, les champignons filamenteux et certains protozoaires ciliés $[8,9,13]$. Elles inhibent la croissance microbienne, d'une manière rapide, irréversible et efficace. En effet, une solution micromolaire de DS inhibe la sporulation et la croissance des semis mycéliens d'Aspergillus fumigatus, Arthroderma simii ou Candida albicans $[9,13]$.

Bien que ces DS présentent une similitude structurale évidente (53 à $94 \%$ ), elles exhibent une différence marquée à inhiber la prolifération microbienne. Cette différence est prononcée vis-à-vis des bactéries [13]. D'ailleurs, ces DS (DS2 et DS3) sont faiblement hémolytiques, et d'une façon surprenante la DS4 entraîne une hémolyse totale à $1 \mathrm{mM}$ $(50 \%$ à $0,5 \mathrm{mM})[8]$.

Dans ce travail, nous nous proposons d'étudier l'effet spermicide de deux DS, en tant que nouvelles structures peptidiques, sur les spermatozoïdes humains. Pour cela nous avons :

- effectué la purification de DS1 et de DS4 ;

- testé in vitro l'activité de ces molécules sur la mobilité et la vitalité des spermatozoïdes humains ;

- étudié l'effet des DS sur les spermatozoïdes humains en fonction du temps et à différentes concentrations ;

- testé l'effet comparatif de ces peptides antibiotiques avec le nonxynol-9, substance de référence utilisée en tant que spermicide ;

- étudié l'effet synergique des DS avec un agent chélateur: Acide éthylène diamine tétra-acétique( EDTA).

\section{MATERIEL ET METHODES}

\section{Les peptides}

Nous avons utilisé comme peptides, la DS1 et la DS4. La synthèse chimique de ces molécules a été effectuée en France dans le cadre d'une collaboration avec l'équipe du laboratoire de bioactivation des peptides à l'Institut Jacques Monod à Paris. II s'agit d'une synthèse en phase solide sur synthétiseur automatique de type Milligen. Cette synthèse nous a permis d'obtenir des séquences peptidiques greffées sur support de résine et donc un produit brut qu'il a fallu traiter.

En Tunisie, nous avons effectué le déblocage et la purification des différentes molécules synthétisées en France. Pour cela, nous avons utilisé la technique classique de purification des peptides, à savoir la Chromatographie Liquide à Haute Performance (HPLC) en phase inverse.

\section{Le sperme}

Le sperme provient de 20 patients suivis pour infertilité du couple au Laboratoire de Cytogénétique et de Biologie de la Reproduction du CHU Farhat Hached de Sousse.

\section{Traitement des peptides à l'état brut}

Tous les peptides que nous avons synthétisés sont, d'une part à l'état brut et d'autres part non débloqués (protégés).

Chaque peptide brut (100 à $150 \mathrm{mg}$ de résine 'R-DS') est mis en suspension dans le cocktail de clivage, selon les proportions suivantes : $5 \mathrm{ml}$ de mélange acide trifluoroacétique(TFA)/Scavengers pour $1 \mathrm{mg}$ de résine-DS pendant 2 heures à température ambiante. La solution est ensuite filtrée sur verre fritté, rincée au TFA et lavée plusieurs fois au Dichlorométhane (DCM). On obtient ainsi une solution sirupeuse que l'on additionne goutte a goutte à une solution de méthyl-terbutyl-ether froid $\left(-20^{\circ} \mathrm{C}\right)$ dans un ou plusieurs tubes 'corex'. Au fur et à mesure de l'addition de la solution sirupeuse, un précipité peptidique se forme au contact de cette solution froide.

On laisse ce précipité se développer une nuit à $4^{\circ} \mathrm{C}$, puis on centrifuge $30 \mathrm{~min}$ à 4000 tours/min. Après élimination de la phase éthérée, l'opération est renouvelée 4 à 5 fois, en rajoutant d'abord un peu d'éther pour bien remettre le précipité en suspension (ultrason, vortex). Au dernier rinçage, on élimine l'éther puis on laisse sécher le peptide 15 min à température ambiante puis une nuit à $60^{\circ} \mathrm{C}$ afin d'éliminer d'éventuels radicaux volatils qui pourraient oxyder les résidus méthionine. On dissout ensuite le peptide dans de l'eau bidistillée et on lyophilise.

Après lyophilisation, le peptide brut est purifié par chromatographie liquide haute performance en phase inverse (HPLC). Nous avons utilisé pour la séparation de ces molécules une chaine de chromatographie (Hewlett Packard série 1050) équipée d'une colonne de compression radiale (type RCM deltapack $C 18$ ) qui peut séparer des quantités importantes de peptides brut (jusqu'à $100 \mathrm{mg}$ ).

Pour la chromatographie analytique, nous avons utilisé la 
même chaîne de chromatographie équipée cette fois d'une

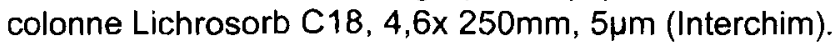

Les peptides ainsi purifiés sont parfaitement stables ; dissous dans de l'eau bidistillée, ils peuvent être conservés à $-20^{\circ} \mathrm{C}$ ou bien à $+4^{\circ} \mathrm{C}$.

\section{Analyse du sperme}

Le recueil du sperme est fait par masturbation, au laboratoire après un délai d'abstinence sexuelle de 3 à 5 jours. L'éjaculât est conservé à $37^{\circ} \mathrm{C}$ pendant $30 \mathrm{~min}$ à l'abri de la lumière jusqu'à liquéfaction du coagulum. L'étude du spermogramme comporte les étapes suivantes :

a) Volume : mesure dans un tube gradué.

b) Viscosité : appréciation de la filance à l'aide d'une pipette Pasteur.

c) $\mathbf{P H}$ : évaluation à l'aide d'un $\mathrm{pH}$ mètre.

d) Numération : Le nombre de spermatozoödes par $\mathrm{ml}$ de sperme est compté à l'aide d'un hémocytomètre (cellule de Thoma) ce qui requiert une dilution variable selon l'estimation initiale de la numération. Le sperme est dilué dans du sérum physiologique formolé à $2 \%$ pour tuer les spermatozoïdes qui sont teintés au bleu de méthylène pour colorer les noyaux [14].

e) Vitalité : estimée par le test de Williams à l'éosine-nigrosine, elle est exprimée en pourcentage de spermatozoïdes vivants.

f) Mobilité : elle est exprimée en pourcentage de spermatozoïdes mobiles et l'analyse qualitative du mouvement précise les pourcentages de spermatozoïdes progressifs et rapides (a), progressifs et lents (b), mobiles sur place (c) ou immobiles (d).

g) Morphologie des spermatozoïdes : le spermocytogramme est réalisé sur un frottis de sperme après coloration au test simplet (Roche). Pour chaque sperme, nous avons déterminé le pourcentage de formes anormales selon la classification de David [6] et l'index des anomalies multiples (IAM) défini par le rapport entre le nombre total d'atypies recensées et le nombre de spermatozoïdes atypiques.

h) Concentration en leucocytes : elle est déterminée par la technique de coloration au benzidine-cyanosine. Le comptage se fait sur cellule de Thoma.

Les valeurs normales retenues sont celles référencées par I'OMS [3] : Volume : 2 à $6 \mathrm{ml} ; \mathrm{pH}: 7,2$ à 7,8; numération $>40$ Millions/éjaculât ; mobilité progressive $(a+b) \geq 50 \%$; vitalité $>30 \%$; formes typiques $\geq 30 \%$; leucocytes $<106 / \mathrm{ml}$.

\section{Traitement du sperme}

La filtration du sperme in vitro a pour but de sélectionner des spermatozoïdes mobiles à morphologie normale, d'éliminer le plasma séminal et les débris cellulaires. Pour cela, nous avons utilisé la technique de centrifugation sur gradient de "Puresperm" ( NidaCon, Göteborg, Suède) à deux couches [15].
Les spermatozoïdes recueillis sont dilués dans un milieu de BM1 (Ellios, Biotek) afin d'obtenir une concentration de 5 à 10 millions $/ \mathrm{ml}$ et placés dans l'étuve $\left(37^{\circ} \mathrm{C}, 5 \% \mathrm{CO} 2\right.$ dans l'air) avant utilisation.

\section{Détermination de l'activité spermicide des derma- septines}

\section{a) Préparation des solutions peptidiques}

Chaque échantillon de DS (DS1 et DS4), pur et sec, est pesé sur une microbalance puis solubilisé dans l'eau bidistillée pour constituer une solution mère de $1 \mathrm{mg} / \mathrm{ml}$. Les dilutions sont effectuées au moment de la réalisation des tests.

\section{b) Estimation de la mobilité}

Pour évaluer l'effet spermicide de la DS, en comparaison avec le Nonoxynol-9(N-9), on prépare trois tubes : le premier renferme $50 \mu \mathrm{l}$ du sperme traité et $50 \mathrm{ml}$ de DS à une concentration bien définie ; le deuxième tube ne contient que $50 \mathrm{ml}$ du sperme traité : il s'agit d'un témoin négatif ; le troisième contient $50 \mathrm{ml}$ de DS ; $50 \mathrm{ml}$ de sperme traité et $0,2 \mathrm{ml}$ de N-9; ce tube servira de contrôle positif.

Ces tubes sont conservés à $37^{\circ} \mathrm{C}$ pour l'estimation de la mobilité et à des intervalles de temps bien précis (2 min, $30 \mathrm{~min}, 60 \mathrm{~min}, 120 \mathrm{~min}$ et $240 \mathrm{~min}$ ).

\section{c) Analyse de la vitalité des spermatozoïdes}

\section{d) Etude de la morphologie des spermatozö̈des \\ e) Test au calcium}

Le calcium active l'ATPase des protéines contractiles et constitue donc un élément essentiel pour la mobilité des spermatozoïdes. Le test au calcium permet de vérifier si l'ajout de calcium peut bloquer l'activité de la dermaseptine.

Nous avons préparé quatre tubes : le premier renferme du sperme traité (témoin); le deuxième contient $50 \mathrm{ml}$ de $\mathrm{Ca}^{2+}(2 \mathrm{mM})+50 \mu \mathrm{l}$ de sperme traité ; le troisième contient $50 \mu l$ de DS4 $+50 \mu l$ de sperme traité ; le quatrième tube contient $50 \mu \mathrm{l}$ de $\mathrm{Ca}^{2+}(2 \mathrm{mM})+50 \mu \mathrm{l}$ de sperme traité +50 $\mu \mathrm{l}$ de DS4.

On laisse les quatre tubes en incubation à $37^{\circ} \mathrm{C}$ pendant une heure, par la suite on fait une estimation de la mobilité des spermatozoïdes.

\section{f) Test à la Pentoxifylline}

La pentoxifylline est un inhibiteur de la phosphodiestérase entrainant l'augmentation de l'AMP cyclique dans le spermatozoïde. Elle est de ce fait, utilisée pour stimuler la mobilité des spermatozoïdes. Le test à la pentoxiphilline permet de vérifier si celle-ci pourrait altérer l'activité de la dermaseptine.

Nous avons procédé de la mêrne manière que pour le test précédent, sauf que nous avons utilisé de la pentoxifylline $(1,5 \mathrm{mg} / \mathrm{ml})$ à la place du calcium.

\section{g) Test à l'éthylène diamine tétra-acétique (EDTA)}

L'EDTA à $0,1 \%$ exerce une activité spermicide en diminuant la concentration en calcium, par la formation de 
complexe EDTA-Ca ${ }^{2+}$. Pour cela nous avons préparé trois tubes : le premier contient $50 \mathrm{ml}$ du sperme traité $+100 \mathrm{ml}$ d'EDTA $(0,1 \%)$; le deuxième tube contient $50 \mathrm{ml}$ du sperme traité $+100 \mathrm{ml} \mathrm{d}$ 'EDTA $+50 \mathrm{ml}$ de DS ; le troisième ne contient que le sperme traité (témoin).

\section{Méthode statistique}

Les test statistiques sont réalisés grâce au programme "SPSS 8.0". La comparaison de plusieurs moyennes a été réalisée par analyse de variance à un seul facteur. La comparaison de deux moyennes a été réalisée par le test $-t$ de Student, ainsi que le test de régression (coefficient de corrélation r).

\section{RESULTATS}

\section{Spermogrammmes et spermocytogrammes}

Tous nos patients ont un spermogramme et un spermocytogramme normaux selon les critères de l'OMS [1].

\section{Purification des peptides par HPLC}

Les profils chromatographiques sur HPLC analytique des deux DS présentés sur la Figure 1 témoignent de la pureté des peptides que nous avons utilisés dans les différents tests d'activité.

\section{Effet des DS sur la mobilité et la vitalité des sperma- tozoïdes}

\section{a) Action de la DS4}

L'analyse de la moitié des échantillons mis en présence de la DS4 montre que à la concentration de $100 \mathrm{mg} / \mathrm{ml}$, la DS4 provoque une inhibition totale et instantanée $(<2 \mathrm{~min})$ de la mobilité des spermatozoïdes (Figure 2). L'effet spermicide est confirmé par l'analyse de la vitalité des spermatozoïdes qui montre qu'à partir de $100 \mathrm{mg} / \mathrm{ml}$, tous les spermatozoïdes sont morts (Figure 3 ). La concentration minimale de DS4 ayant une activité spermicide totale et instantanée : EC100 DS4 est donc égale à $100 \mathrm{mg} / \mathrm{ml}$. A la concentration de $50 \mu \mathrm{g} / \mathrm{ml}$, elle altère la mobilité spermatique de façon significative après $30 \mathrm{~min}$ d'incubation par rapport à des concentrations plus faibles $(p=0,013)$ et la comparaison des pourcentages moyens des spermatozoïdes morts après 2 et 30 min d'incubation trouve une différence très significative $(p=0,003)$ (Figure 3 ).

A partir de $25 \mathrm{mg} / \mathrm{ml}$, la DS4 ne devient efficace qu'après 1 heure d'incubation par rapport à des temps d'incubation plus courts $(p=0,001)$. Son effet se prolonge jusqu'à 4 heures d'incubation de façon lente et la comparaison de la mobilité moyenne spermatique après 1 heure et 4 heures d'incubation montre une différence faiblement significative $(p=0,038)$ (Figure 2).

L'analyse de la vitalité spermatique après différents temps d'incubation montre une altération très significative après une heure d'incubation par rapport à des temps plus courts $(p=0,007)$ et le nombre de spermatozoïdes morts continue à augmenter jusqu'à 4 heures d'incubation ( $1 \mathrm{~h}$ vs $4 \mathrm{~h}$, $\mathrm{p}=0,011$ ) (Figure 3).

La DS4 demeure active contre la mobilité de même à de faibles concentrations $(5 \mathrm{mg} / \mathrm{ml})$. Son effet devient significatif après une heure $(p=0,047)$ et total après 4 heures d'incubation (Figure 2).

\section{b) Action de la DS1}

La DS1 ne semble pas avoir le même degré d'activité spermicide que la DS4. En effet, à partir de $200 \mathrm{mg} / \mathrm{ml}$, elle provoque la mort totale et instantanée $(<2 \mathrm{~min}$ ) de tous les spermatozoïdes (Figure 4). La concentration minimale de DS1 ayant une activité spermicide totale et instantanée : EC100 DS1 est donc égale à $200 \mathrm{mg} / \mathrm{ml}$.

A $125 \mathrm{mg} / \mathrm{ml}$ et après 2 heures d'incubation, elle altère significativement le pourcentage moyen des spermatozoïdes morts par rapport à des temps plus courts $(p=0,008)$ et après 4 heures d'incubation, elle entraîne la mort totale de tous les spermatozoïdes (Figure 4).

Par contre, à $100 \mathrm{mg} / \mathrm{ml}$, elle n'altère significativement la mobilité spermatique qu'après 4 heures d'incubation par rapport à des concentrations plus élevées $(p=0,001$ ) (Figure 5). L'analyse de la vitalité confirme ces résultats avec une différence significative entre le pourcentage moyen des spermatozoïdes morts après 30 et 240 min d'incubation $(p=0,001)$.

La DS1 à $50 \mathrm{mg} / \mathrm{ml}$ présente presque les mêmes effets sur la mobilité et la vitalité spermatique qu'à $100 \mathrm{mg} / \mathrm{ml}$. Elle devient inefficace à partir de $25 \mathrm{mg} / \mathrm{ml}$, en effet après 4 heures d'incubation, elle entraîne une augmentation non significative du nombre de spermatozoïdes morts et une faible diminution de la mobilité spermatique (Figure 5).

\section{c) En présence des DS et de l'EDTA}

L'association des DS et de l'EDTA en présence des spermatozoïdes se traduit par une importante diminution de leur mobilité : l'EDTA à une concentration de $0,1 \%$ montre un effet spermicide modéré, témoignant ainsi d'une activité spermicide moyenne ; la DS4 à une concentration de 5 $\mathrm{mg} / \mathrm{ml}$ entraîne l'inhibition de la moitié des spermatozoïdes en 30 minutes alors que l'addition de l'EDTA à $0,1 \%$ cause une inhibition totale de la mobilité des spermatozoïdes dans les 30 minutes qui suivent (Figure 6). Ces résultats sont en faveur de la présence d'un effet synergique entre la DS4 et l'EDTA. La DS1 présente aussi un effet synergique avec I'EDTA $(0,1 \%)$ (Tableau 1). Cependant, cet effet reste moins important que pour la DS4.

\section{d) En présence des $D S$ et de la pentoxifylline}

D'abord nous avons étudié l'effet de la pentoxifylline seule sur la mobilité et la vitalité des spermatozoïdes et après 4 heures d'analyse, nous n'avons trouvé aucun effet significatif sur ces paramètres. Dans un deuxième temps nous avons ajouté de la DS4 à $50 \mathrm{mg} / \mathrm{ml}$ en présence de la pentoxifylline et des spermatozoïdes (Figure 7) et nous n'avons trouvé aucun effet synergique sur l'activité spermicide des DS et ce, quelque soit le temps d'incubation.

\section{e) En présence des DS et du calcium}

Le but de cette expérience est de vérifier si la DS "tue" les spermatozoïdes en "piégeant" le calcium intracellulaire. Les résultats ont montré que l'addition du calcium même à 


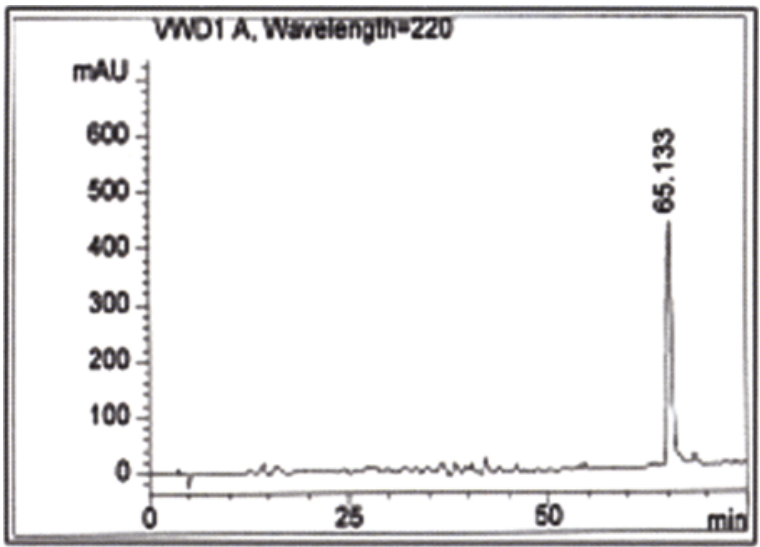

DS1

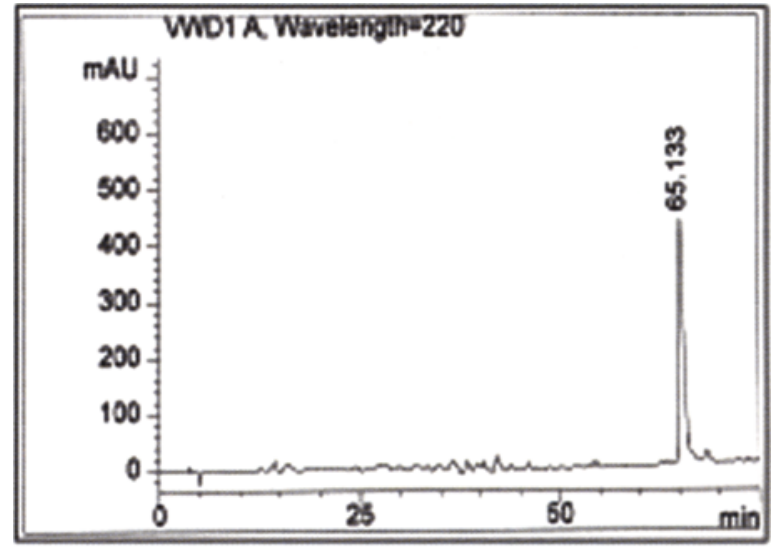

DS4

Figure 1 : Profil chromatographique en HPLC analytique des DS purifiées (DS1 et DS4).

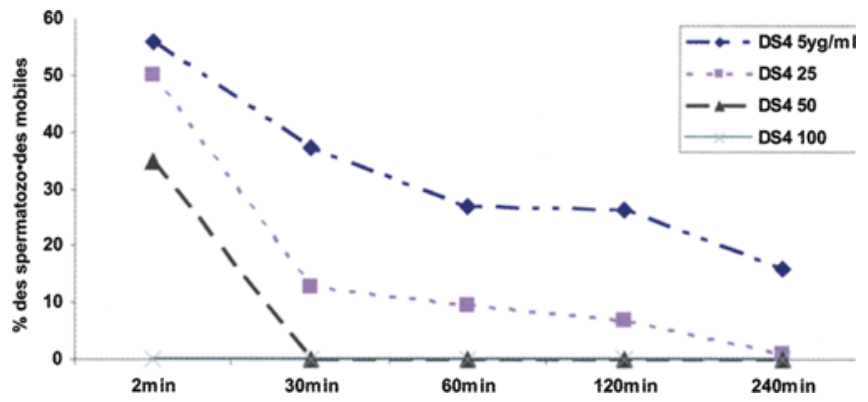

Figure 2 : Evolution des pourcentages moyens des spermatozoïdes mobiles en fonction des concentrations de la DS4 (en $\mu \mathrm{g} / \mathrm{mI}$ ) et des temps d'incubation.

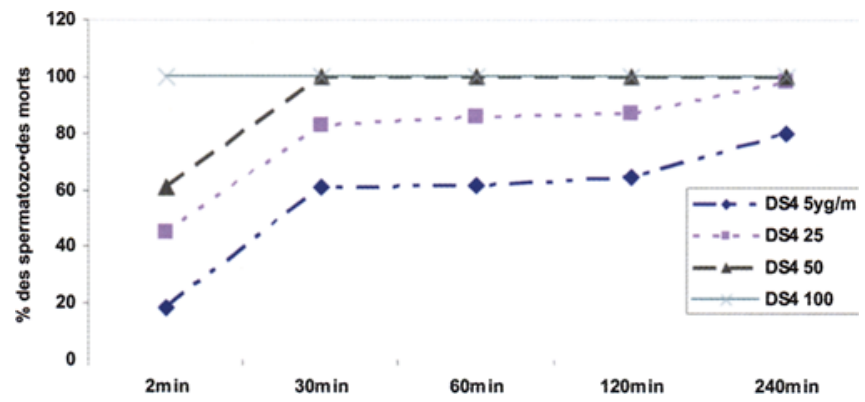

Figure 3 : Evolution des pourcentages moyens des spermatozoïdes morts en fonction des concentrations de la DS4 (en $\mu \mathrm{g} / \mathrm{ml})$ et des temps d'incubation.

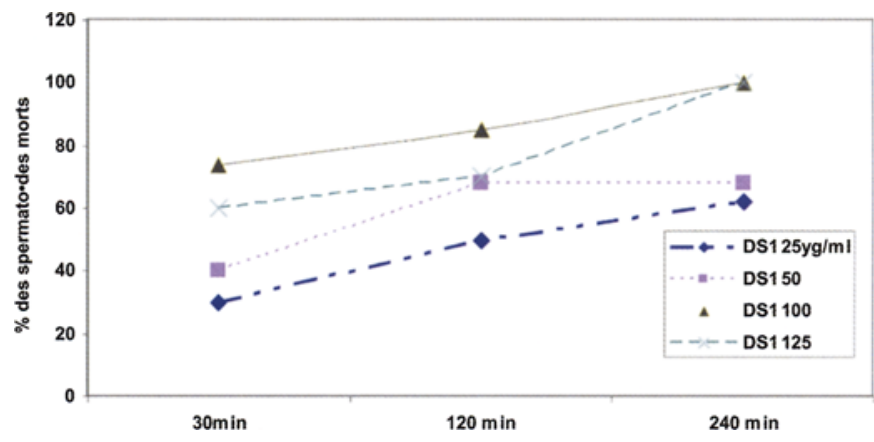

Figure 4 : Evolution des pourcentages moyens des spermatozoïdes morts en fonction des concentrations de la DS1 (en $\mu \mathrm{g} / \mathrm{ml}$ ) et des temps d'incubation.

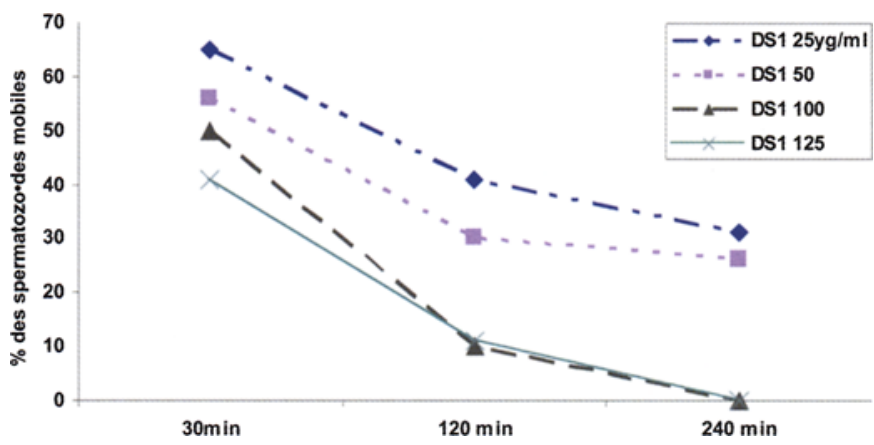

Figure 5 : Evolution des pourcentages moyens des spermatozoïdes mobiles en fonction des concentrations de la DS1 (en $\mu \mathrm{g} / \mathrm{ml}$ ) et des temps d'incubation. 


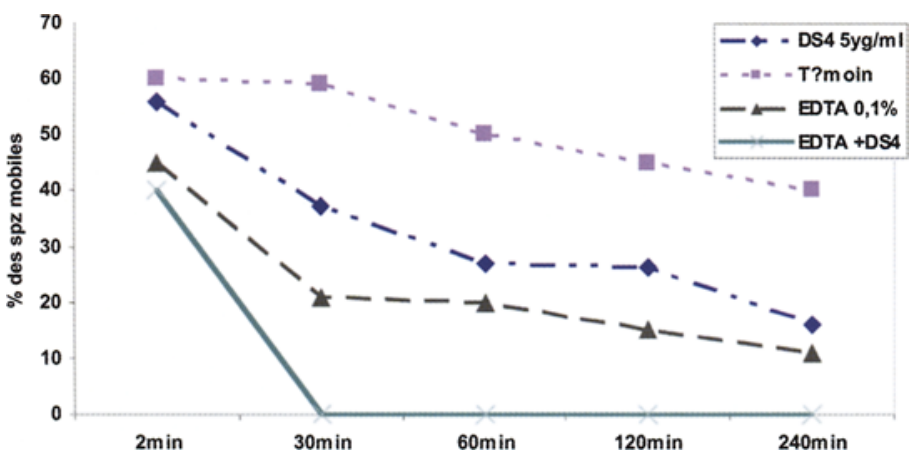

Figure 6 : Effet synergique de I'EDTA et de la DS4 sur la mobilité des spermatozoïdes.

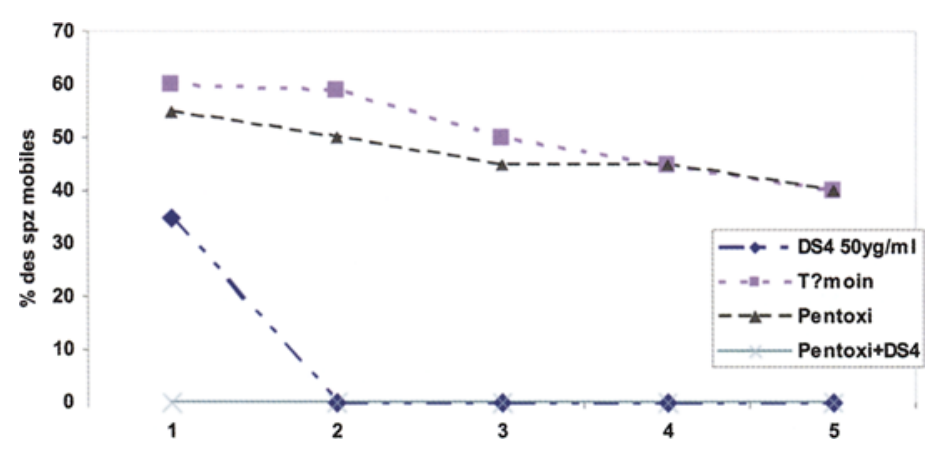

Figure 7 : Effet synergique de la pentoxifylline et de la DS4 sur la mobilité des spermatozoïdes.

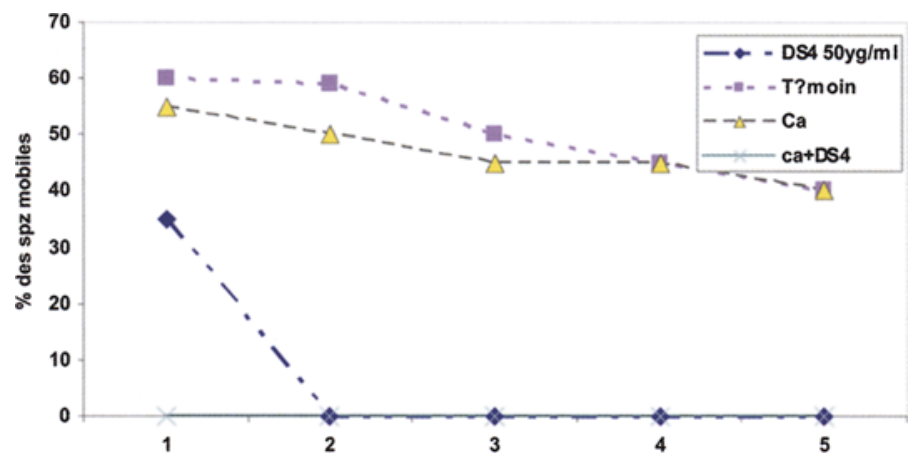

Figure 8 : Effet synergique du calium et de la DS4 sur la mobilité des spermatozoïdes.
Tableau 1 : Effet synergique de I'EDTA et de la DS1 sur la mobilité des spermatozoïdes après 30 minutes d'incubation.

$\begin{array}{ccc}\text { Concentration de } & \text { Spermatozoïdes mobiles à } 30 \text { min (\%) } \\ \text { DS1 } & \text { DS1 } & \text { DS1+EDTA 0,1\% }\end{array}$

\begin{tabular}{ccc}
\hline 25 & 65 & 40 \\
50 & 56 & 36 \\
100 & 50 & 22 \\
125 & 44 & 18 \\
200 & 0 & 0 \\
250 & 0 & 0 \\
\hline
\end{tabular}

une concentration élevé ( $2 \mathrm{mM})$ n'a aucun effet sur la mobilité et la vitalité des spermatozoïdes en présence de la DS4 (50 mg/ml) et ce, quelque soit le temps d'incubation (Figure 8).

\section{DISCUSSION}

\section{Les DS sont actives contre les spermatozoïdes humains}

La DS1 et la DS4 montrent une importante activité spermicide contre les spermatozoïdes humains. En effet, on s'aperçoit qu'à des concentrations micromolaires, ces DS entraînent une inhibition totale de la mobilité des spermatozoïdes après un temps d'incubation relativement court (30 min) comparativement à d'autres substances.

Cette activité spermicide des DS, que nous avons montrée pour la première fois, a été confirmée par le test de vitalité, qui a montré qu'en présence de ces peptides, tous les spermatozoïdes meurent à plus ou moins long terme. Cependant, en augmentant leur concentration, les DS finissent toutes par devenir actives contre les spermatozoïdes en un temps très bref.

L'importance des DS en tant que spermicides réside dans le fait qu'elles exercent leur activité à une concentration relativement faible $(100 \mu \mathrm{g} / \mathrm{ml})$ et inférieure à celle du nonoxynol-9.

Le nonoxynol-9 à $5 \mu \mathrm{g} / \mathrm{ml}$ n'a aucun effet sur la mobilité des spermatozoïdes même après une période d'incubation dépassant les 4 heures [7], alors qu'à cette même concentration, la DS4 entraîne l'inhibition de la mobilité de plus de $80 \%$ des spermatozoïdes après seulement deux heures d'incubation avec une diminution significative du pourcentage moyen des spermatozoïdes à trajectoire directe.

La magainine, un antimicrobien de structure peptidique, possède aussi une activité spermicide. Etant le prototype des peptides très fortement cationiques, la magainine entraîne l'inhibition totale de la mobilité des spermatozoïdes à $200 \mu \mathrm{g} / \mathrm{ml}$ et ce après deux heures d'incubation [16]. Comparativement, la DS4 est douée d'une activité spermicide plus importante puisqu'à la même concentration que 
la magainine, la DS4 a un effet spermicide instantané. De ce fait, on peut conclure que la DS et la magainine, bien qu'elles appartiennent à la même famille (Peptides en hélice $\alpha$ ), présentent une différence notable au niveau de leur activité spermicide, activité qui varie donc d'un peptide à un autre.

Quand on compare la DS4 comme agent spermicide à deux autres produits testés pour leur activité spermicide, le VDC (vanadocenes) et le WHI-7 (dérivé de la Zidovudine), on trouve que ces dernières substances inhibent la mobilité des spermatozoïdes à des concentrations élevées, respectivement $100 \mu \mathrm{M}$ et $1000 \mu \mathrm{M}$ [5].

Ces premiers résultats concernant le développement de nouveaux agents spermicides sont très encourageants et on peut raisonnablement s'attendre à ce que l'utilisation de ces nouvelles structures peptidiques se développe dans les années à venir.

\section{Les DS ne sont pas forcément actives de la même manière}

Nous avons montré précédemment, que la DS4 et la DS1 sont toutes les deux actives contre les spermatozoïdes mais à différentes doses. La DS1 à une concentration élevée $(125 \mu \mathrm{g} / \mathrm{ml})$, a un effet spermicide très faible après quatre heures d'incubation; à cette même concentration, la DS4 a un effet spermicide instantané.

On peut dégager de ces observations que l'efficacité des DS vis-à-vis des spermatozoïdes varie d'un peptide à un autre. Ceci renforce l'idée que les DS, bien que présentant une homologie structurale évidente, exhibent une différence marquée au niveau de leur activité qui réside certainement au niveau de certains paramètres tels le caractère cationique, le caractère hémolytique, l'hélicité de la molécule et l'index d'hydropathie ou d'hydrophobicité.

\section{Est-ce que l'activité spermicide de la DS4 est plus importante que celle de la DS1?}

Si l'on compare l'activité spermicide pour chacune des deux DS, on constate que la DS4 possède de loin l'activité spermicide la plus importante vues les concentrations inhibitrices ou effectives qu'elle présente.

Les résultats trouvés et présentés auparavant indiquent que la DS4 entraîne l'inhibition totale de la mobilité et de la vitalité des spermatozoïdes ; en effet, $100 \%$ des spermatozoïdes sont morts à une concentration de $50 \mu \mathrm{g} / \mathrm{ml}$ en 30 minutes, par contre elle reste moins efficace à $25 \mu \mathrm{g} / \mathrm{ml}$. On peut donc passer à $100 \%$ d'inhibition de la mobilité en doublant la concentration du peptide.

Cependant, la DS4 montre une cytotoxicité élevée d'après les résultats trouvés, mais cette particularité reste controversée. En effet, la corrélation entre l'activité spermicide et la cytotoxicité n'est pas vérifiée. Ceci est illustré dans l'exemple de la DS1 qui n'est pas très active contre les spermatozoïdes et qui présente pourtant une toxicité assez élevée comparable à celle de la DS4.

De plus, la DS4 présente non seulement une cytotoxicité relative, mais aussi une activité hémolytique très importante. En effet, il a été démontré que la DS4 est hémolytique avec $100 \%$ de lyse cellulaire à $1 \mu \mathrm{M}$. Cette activité semble être liée essentiellement au degré d'hydrophobicité du domaine $\mathrm{N}$-terminal du peptide en question. En effet, plus le nombre de résidus hydrophobes est important, plus l'activité hémolytique est marquée.

Cette particularité ne peut limiter le spectre d'activité des DS et en particulier l'activité spermicide, parce qu' il s'est avéré que l'activité hémolytique peut être abolie en diminuant l'hydrophobicité.

Une étude systématique approfondie des relations structure-fonction de la DS4 peut être envisagée comme perspective d'avenir : il s'agit de faire varier la charge globale du peptide ou d'intervenir sur l'hélicité et l'amphipathie et de corréler ces paramètres structuraux avec l'activité biologique.

\section{Les DS et I'EDTA agissent en synergie}

Au cours de ce travail, nous avons pu constater que l'activité spermicide des DS, et en particulier la DS4, était fortement augmentée en présence d'un agent chélateur tel que I'EDTA. En effet, la DS4 montre une action synergique avec l'EDTA $(0,1 \%)$ qui amplifie considérablement son activité spermicide.

Si le peptide présente individuellement une action efficace contre les spermatozoïdes à une concentration de 50 $\mu \mathrm{g} / \mathrm{ml}$, sa présence simultanée avec l'EDTA permet d'augmenter son efficacité et d'obtenir une action spermicide instantanée, alors que l'EDTA à $0,1 \%$ présente une activité spermicide relativement faible.

Ce phénomène de synergie entre I'EDTA et la DS4, s'explique par le fait que cet agent chélateur va renforcer l'activité spermicide de la DS4 grâce à son action sur le calcium. En fait, I'EDTA entraîne la diminution du taux intracellulaire du calcium via la formation du complexe (Ca2+EDTA), sachant que le calcium est un élément clé dans la mobilité des spermatozoïdes.

D'ailleurs, quand on compare l'effet synergique entre la DS4 et I'EDTA en présence du nonoxynol-9, on remarque que cette dernière est beaucoup moins importante. En effet, pour le nonoxynol-9 en présence de l'EDTA $(0,1 \%)$, sa concentration effective passe de $100 \mu \mathrm{g} / \mathrm{ml}$ à $66,4 \mu \mathrm{g} / \mathrm{ml}$ [10], alors que pour la DS4 en présence de l'EDTA, l'EC100 passe de $50 \mu \mathrm{g} / \mathrm{ml}$ à $5 \mu \mathrm{g} / \mathrm{ml}$. Ceci reflète la forte synergie entre la DS (DS4) et cet agent chélateur qui s'explique par la formation de complexes (EDTA-Ca 2+).

\section{Pourquoi cette sélectivité des DS ?}

Malgré l'homologie des séquences assez importante des DS, nous pouvons remarquer d'emblée qu'elles présentent un potentiel d'activité similaire mais non identique ; autrement dit, les DS (à des faibles concentrations) sont plutôt sélectives vis-à-vis des spermatozoïdes. Cependant, en augmentant leurs concentrations, elles finissent par être toutes actives contre ces cellules. 
Nous pouvons dégager de ces observations une propriété caractéristique des DS qui pourrait montrer leur profil d'activité hétérogène, c'est à dire leur capacité différentielle à cibler spécifiquement les spermatozoïdes.

\section{Comment ces DS agissent-elles sur les spermatozoï-} des?

Bien que le mécanisme d'action de certains spermicides tels que le nonoxynol-9 soit bien défini, celui de certaines molécules peptidiques à l'instar des DS reste encore à élucider.

En effet, l'inhibition de la mobilité du spermatozoïde par la DS4 est rapide, voire même instantanée pour certaines concentrations et ceci peut être expliqué par l'affinité que présentent ces structures amphiphiles aux bicouches lipidiques.

Ainsi, on sait que ces peptides antimicrobiens structurés en hélice $\alpha$ amphipatique, semblent avoir des propriétés membranolytiques marquées vis-à vis de leurs cibles. Ceci perturbe l'organisation des bicouches lipidiques via la formation de canaux ioniques, entraînant ainsi une dissipation du potentiel membranaire. II a été démontré que cette interaction peptide-membrane est différente avec les cellules des mammifères, dont les membranes sont peu perméables. Cependant des exceptions existent et des études récentes ont montré que certains peptides à l'instar des magainines présentent une activité contre des cellules eucaryotiques et en particulier les spermatozoïdes.

On peut ainsi imaginer que l'interaction peptide-spermatozoide existe, qu'elle est moins accentuée que les autres interactions, mais qu'elle est suffisante pour induire une perméabilité au niveau membranaire, réaliser un déséquilibre ionique et entraîner par la suite la mort des spermatozoïdes.

Enfin, nous avons pu montrer que les DS ne présentent aucun effet sur les éléments clés qui interviennent dans le mouvement du spermatozoïde tels que la pentoxifylline et le calcium. Nous avons constaté par la suite que leur addition ne bloque pas l'effet spermicide des DS. Ceci renforce l'idée qu'il y a une interaction peptide-spermatozoïde au niveau membranaire, cependant d'autres études complémentaires se rapportant à l'ultrastructure des spermatozoïdes sont nécessaires pour mieux comprendre le mécanisme de l'activité spermicide des DS et son utilité certaine en tant que spermicide et antimicrobien à la fois.

\section{CONCLUSION}

L'analyse de l'activité des DS, nous a permis de montrer pour la première fois que ces peptides :

- sont actifs contre les spermatozoïdes humains ;

- ont un spectre d'activité spermicide comparable sinon meilleur que celui du nonoxynol-9, qui représente actuellement le spermicide de choix le plus utilisé ;

- ont un mécanisme d'action différent de celui du nonoxynol-9 ;
- entrainent l'inhibition de la mobilité des spermatozoïdes humains après un temps d'incubation relativement court, suggérant ainsi un mode d'interaction direct du peptide avec la membrane du spermatozoïde ;

- ont une parfaite action synergique avec d'autres spermicides, tels que les agents chélateurs (EDTA).

Mais il est connu que la DS4 présente une activité hémolytique élevée $(100 \%$ de lyse à $1 \mu \mathrm{M})$ [8] ce qui limitera son éventuelle application thérapeutique en tant que spermicide. Ceci nous incite à continuer les efforts orientés vers la synthèse d'analogues de structure en tant que candidats potentiels pour la conception de nouvelles substances efficaces, dénuées de cytotoxicité, ayant une bioactivité élevée et qui puissent être utilisées à visée contraceptive.

Il est donc nécessaire d'approfondir ces études en :

- évaluant l'innocuité autant que l'activité spermicide des autres DS (DS2, DS3 et DS5) afin de comparer leurs efficacités sur les spermatozoïdes ;

- étudiant des molécules plus raccourcies des DS. De telles mini structures permettraient d'élucider leur mécanisme d'action au niveau moléculaire, ce qui devrait ouvrir la voie à une nouvelle génération de molécules à spectre d'action large et variée utilisables comme nouveaux spermicides ;

- testant l'action de ces nouvelles structures sur d'autres germes pathogènes responsables des infections au niveau du tractus utéro-vaginal ainsi que sur le VIH [2].

\section{REFERENCES}

1. AUGER J., EUSTACHE F. : Standardisation de la classification morphologique des spermatozoides humains selon la méthode de David modifiée. Andrologie, 2000, 4 : 358-373.

2. BELAID A., AOUNI M., KHELIFA R., TRABELSI A., JEMMALI M., HANI K. : In vitro antiviral ctivity of Dermaseptins against Herpes Simplex Virus Type 1. J. Med. Virol, 2002, $66: 229-$ 234.

3. CHARPENTIER S., AMICHE M., MESTER J., et al. : Structure, synthesis, and molecular cloning of dermaseptins $B$, a family of skin peptide antibiotics. J. Biol. Chem., 1998, 273 : 14680-14697.

4. COLLIER F., LETOMBE B. : Contraception naturelle et chimique chez la femme. Encyclopédie Médico-Chirurgicale, 1998, 738-A-11.

5. D'CRUZ O.J., DONG Y., UCKUN F.M. : Spermicidal activity of oxavanadium (IV) complexesof1,10-phenanthroline,2,2-Bipyridyl,5'-Bromo-2'-hydroxyalkoxybenzyl -oxopyrimidine are dual function spermicide with potent anti-human immunodeficency virus activity. Biol. Reprod.,1999A, 60 : 1419-1428.

6. DAVID G., BISSON J.P., CZYGLIK F. et al. : Anomalies morphologiques du spermatozoïde humain, proposition pour un système de classification. J. Gyn. Obstet. Biol. Reprod., 1975,4 suppl $1: 17-36$.

7. DAVID R., WHITE E., CLARSON J.C., RATNASOORIYA W.D., AITKEN J. : Complementary effects of propanol and 
nonoxynol-9 upon human sperm motility. Contraception, 1995, $52: 241-247$.

8. FEDER R., DAGPON A., MOR A. : Structure-activity relationship study of antimicrobial dermaseptin $\mathrm{S} 4$ showing the consequences of peptide oligpomerization on selective cytotoxicity. J. Biol. Chem., 2000, $275:$ 4230-4238

9. KRUGLIAK M., FEDER R., ZOLOTAREV V.Y. et al. : Antimalarial activities of Dermaseptin $\$ 4$ derivates. Antimicrobial agents and chemotherapy, 2000, $44: 2442-2451$.

10. LEE C.H., BAGDON R., CHIEN Y.W. : Comparative in vitro spermicidal activity of chelating agents and synergetic effect with nonoxynol-9 on human sperm functionnality. J. Pharm. Sci., 1996, $85:$ 91-95.

11. MOR A., DELFOUR A., NICOLAS P. : Identification of D-Alanine-containing polypeptide precursor for the peptide opoid, dermophin. J. Biol. Chem., 1991B, $266: 6264-6270$.

12. MOR A., NGUYEN V.H., DELFOUR A., MIGLIORE-SAMOUR D., NICOLAS P. : Isolation, aminoacide sequence and synthesis of dermaseptin, 3 novel antimicrobial peptide of amphitian skin. Biochemistry, 1991A, $30: 8824-8830$.

13. MOR A., ROUFFAUD M.A., MONTAGNE J.J., NGUYEU V.H., NICOLAS P. : Natural and synthetic dermaseptins : in vitro large spectrum antimicrobial peptides. J. Mycol. Med., 1993, 3: 137-143.

14. PINATEL M.C. : Spermogramme : Technique de réalisation. Ann. Biol. Clin., 1985, $43: 49-53$.

15. SÖDERLUND B., LUNDIN K. : The use of silane-coated silica particules for density gradient centrifugation in-vitro fertilisation. Hum. .Reprod., 2000, $15: 857-860$.

16. WOJEIK C., SAWACKI W., RARIANOUSKI P., BENCHAIB M., CZYBA J.C., GUERIN J.F. : Cyclodextrin entrances spermicidal effets of magainin-2-amide. Contraception, 2000, 61 : 99103.

17. ZAVOS P.M., CORREA J.R., ZARMAKOUPIS-ZAVOS P.N. : Assessement of a tablet drug delivery system incorporating nonoxynol-9 coprecipitated with polyvinylpyrrolidone in preventing the onset of pregnancy in rabbits. Fertil. Steril., 1998, $69: 768-773$.

Manuscrit reçu : mai 2004 ; accepté janvier 2005

\section{ABSTRACT}

In vitro effects of dermaseptins on sperm characteristics

Mounir AJINA, Amira ZAIRI, Soumaya MOUGOU, Samira IBALAA, Radhouène NAIEFER, Ghaya MERDASSI, Mariem MEHDI,Khaled EL HENI, Ali SAAD

The spermicidal efficacy of synthetic peptides, dermaseptin (DS1) and (DS4), was studied under in vitro conditions using human spermatozoa. The data showed that sperm motility was inhibited with various concentrations of dermaseptins at different intervals ranging from 2 to $240 \mathrm{~min}$. The effective $100 \%$ inhibitory concentration (EC100) of DS4 sperm immobilization assay was equal to $50 \mathrm{mg} / \mathrm{ml}$ at $30 \mathrm{~min}$, while the EC 100 of DS1 was equal to $100 \mathrm{mg} / \mathrm{ml}$. The presence of $0.1 \%$ of chelating agent, EDTA, reduced the EC100 of DS4 to $5 \mathrm{mg} / \mathrm{ml}$, while less than a twofold enhancement in DS 1 activity was observed in combination with EDTA.

The action of dermaseptins on sperm motility was observed to be dose-dependent. Addition of pentoxifylline, which is known to enhance sperm motility, and $\mathrm{Ca}^{2+}$, which is a key element for sperm movement, did not prevent the spermicidal action of dermaseptins.

Key words: dermaseptin, spermicide, spermatozoa 